\title{
Review of: "Guiding ATR and PARP inhibitor combinations with chemogenomic screens"
}

\author{
Alejandra Bruna
}

Potential competing interests: The author(s) declared that no potential competing interests exist.

In this manuscript the authors perform a forward functional CRISPR screen to identify genetic biomarkers of synthetic lethality to the combination of PARPi and ATRi . RNAseH2, RAD51 are amongst the genetic hits identified that hypersensitize cells to this combination therapy. In addition, the authors clearly show tumour cells positive for ATL are hypersensitive to ATRi/PARPi combination. Significantly, the authors design a new drug scheduling strategy for maximising the efficacy and lowering the toxicity of this approach.

THe relevance of this data also relies on the connection to their clinical trial using RT3500.

Synthetic lethality based on DDR factors remains a hot topic. I would suggest they highlight the novelty of their approach already in the abstract (for example the identification of multiple mechanisms of ATRi/PARPi synergy....). In addition, a BRAC1-/- would act as a good control/benchmark.

Why would the authors choose a retinal pigment cell line in their original CRISPR screen? My major comment to the manuscript is the diversity of cell lines used without rationalizing the choice. Although I understand the strong implications of finding a wide range synthetic lethality approach, many previous observations suggest cell type dependent molecular mechanisms of drug responses. The heterogeneity of drug responses could also be due to non-genetic factors and therefore, the use of different tissue of origin cell lines might add an extra layer of confounding factors to the data. This might be relevant in later stages of the project though.

I suspect from 155-168 are part of the discussion?.

Did the replication catastrophe also occurred in ALT- and/or RAD51 deficient cells?

The observed correlation between ALT+ and increased sensitivity is interesting, yet will required further mechanistic and experimental observations . 